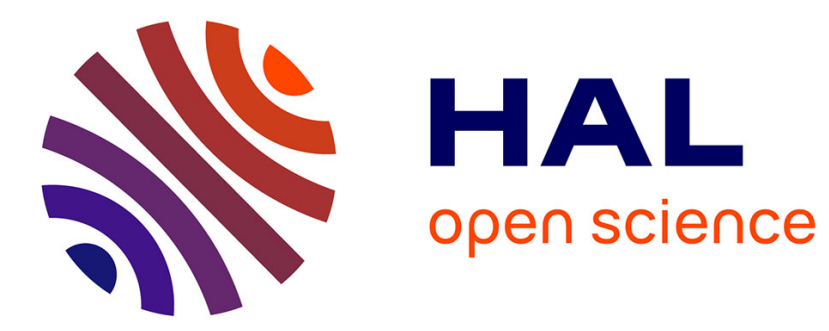

\title{
Blur identification in image processing
}

\author{
Jérôme da Rugna, Hubert Konik
}

\section{To cite this version:}

Jérôme da Rugna, Hubert Konik. Blur identification in image processing. IJCNN 2006, Jul 2006 , Vancouver, Canada. pp.2536-2541, 10.1109/IJCNN.2006.247106 • ujm-00124897

\section{HAL Id: ujm-00124897 https://hal-ujm.archives-ouvertes.fr/ujm-00124897}

Submitted on 16 Jan 2007

HAL is a multi-disciplinary open access archive for the deposit and dissemination of scientific research documents, whether they are published or not. The documents may come from teaching and research institutions in France or abroad, or from public or private research centers.
L'archive ouverte pluridisciplinaire HAL, est destinée au dépôt et à la diffusion de documents scientifiques de niveau recherche, publiés ou non, émanant des établissements d'enseignement et de recherche français ou étrangers, des laboratoires publics ou privés. 


\title{
Blur identification in image processing
}

\author{
Jérôme Da Rugna and Hubert Konik
}

\begin{abstract}
The aim of this study is to achieve a blur identification task in still images. In fact, in photographic camera, the optical lenses may be set in a way to clearly distinct two areas in the image : the blurry one and the non blurry one. An automatic segmentation coupled to specific descriptors allow first to describe any region of the image. Then, a supervised learning processes permits to build a classifier able to decide for each unknown region the label "Blurry" or "Sharp". We discuss here precisely the overall process, from the objective choice of the segmentation algorithm to the presentation of the different introduced descriptors. Finally, some results are presented validating such an approach.
\end{abstract}

\section{INTRODUCTION}

Considering classical holiday photography or portraits, the blurry regions generally represent the background, otherwise speaking regions of no interest for the viewer. On the contrary, the sharp area can normally be seen as the interest part of the global image, at least for the one who took the picture... In fact, if a photographer decides to focus only on some objects in an image, he certainly considers that the rest of the image is not of the prime interest. Let consider the examples presented in figure 1, where objects of attention are clearly salient. Let first introduce classical models of blur and depth computing usually used talking about such a tak.

\section{A. Point Spread Function}

Classically, the blur is modeled in image processing as :

$$
g(x)=(h \star f)(x)
$$

with $x$ a pixel, $g$ the blurred image, $f$ the unknown sharp image and $h$ the point spread function, noted PSF[1] $]^{1}$. In the case of image restoration, the blur is unwanted and has to be compensated. Then, the PSF has to be evaluated [2] before being inverted so that the original sharp image can be reconstructed. Many estimators evaluate the PSF through a unique numerical value : "from sharp to really blur", but it is not casual to consider a threshold $T$ like

$$
\text { if } P S F<T \text { then Sharp else Blur }
$$

Nevertheless, PSF estimator gives more or less only an information of smooth around a point but not any information inside a specific zone.

Jérôme Da Rugna and Hubert Konik are with the Laboratoire LIGIV, Université Jean Monnet, 18 rue B. Lauras, 42000 Saint-Étienne, France (email: \{darugna,konik\}@ligiv.org)

${ }^{1}$ The symbol $\star$ represents the convolution operator

\section{B. Depth from focusing}

Evaluating depth in image is important in many applications. Depth from focus and depth from defocus techniques approaches[3], using a bunch of images of the same scene, propose to evaluate the depth of any elements contained in the image. This bunch of images is obtained by combining different optic properties of the camera, like the focus position. A restriction of these approaches is to be efficient specially on indoor scene, where infinite depths are not present[4].

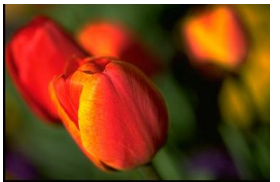

(a)

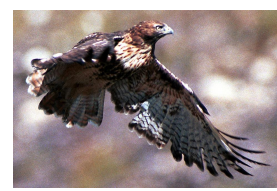

(d)

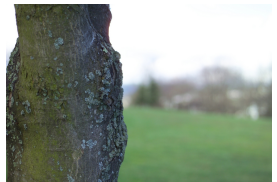

(b)

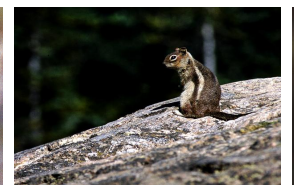

(e)

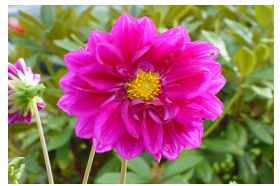

(c)

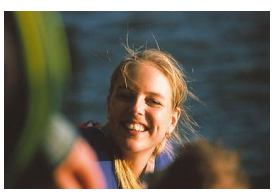

(f)
Fig. 1. Some blurry images.

\section{Context and aim}

After having resumed principal approaches used to blur estimation in computer vision, let illustrate in figure 2 the more precisely content of this study extracting from an image the sharp one and the blurry one. Then, as presented before, PSF estimator approaches are not directly usable and depth from focusing techniques are excluded as it needs several images of the same scene. Thus, we propose a new approach, for blur identification, first based on an image segmentation. This approach mixes as well pixel and region processes, like PSF estimator, to categorize Blurry / Non Blurry.

Before describing the identification task let first specify the image domain covered by this work. In deed, several kinds of blur are possible in an image, issue from optical lenses, motion or any image modification with a specific software. Nevertheless, we consider only optical blur, id est the classical still image where the object of attention is salient. Finally, images we deal with may be described as follow:

- made by a camera and gone through few image processing modifications.

- containing sharp zone and maybe a blurry one (camera focus was made on a specific zone of the scene). We do 
not restrict the position of the focused zone and this zone may be composed of several not connected areas.

- not containing any other kind of blur.
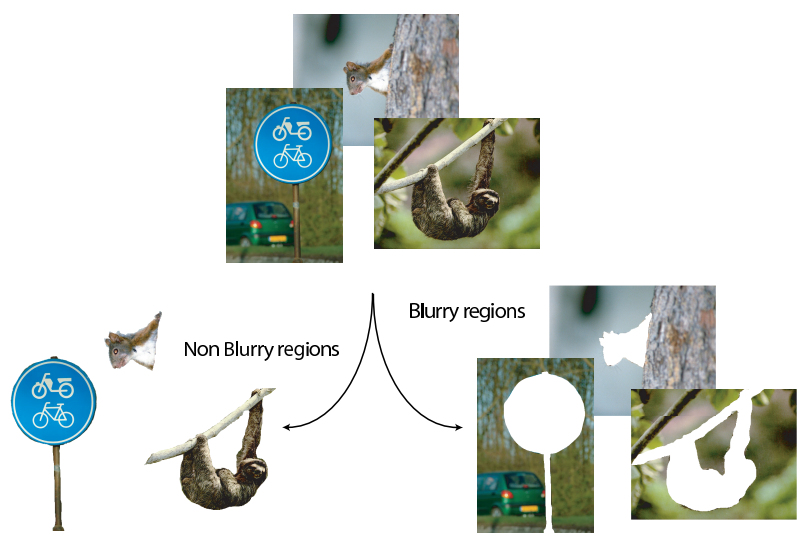

Fig. 2. Blur identification

\section{OVERALL PROCESS}

To reach our goal, two main approaches may be considered

- Evaluating blur function at pixel level.

- Evaluating blur function globally for each region.

In this study, we present a schema that follows the region approach as in fact, evaluating Blur on each pixels is useful mainly on restoration cases. Nonetheless, to identify blurry zone in an image,we assume that it requires a region approach directly based on human blur perception that does not consider point properties but as zone aspects[5].

Before developing more precisely image processing algorithms, let present the learning process and the identification task.

\section{A. Learning process}

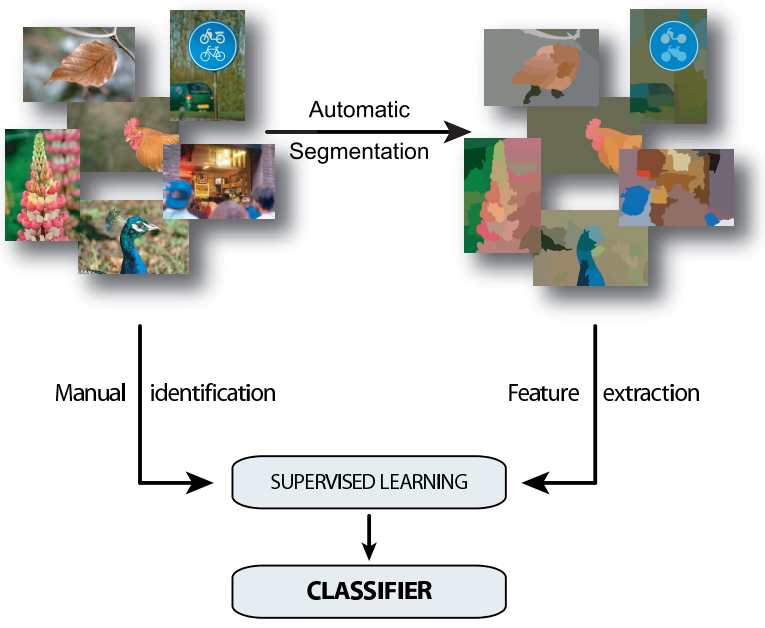

Fig. 3. The learning process.
Let first describe the training task presented in figure 3 where a training set of images is considered. Each of them has previously been selected according to the specifications previously defined in the introduction. For each image, we extract through an expertise two areas (the blurry one and the non blurry one). This identification on the training set is of course subjective as it was made using human judgement, in our case by image analysis specialists and amateur photographs. We may note that this decomposition is made without considering any segmentation : the expert has to draw using a precise pencil the two different zones.

Thus, considering the segmented image and its manual annotation, it is possible to specify for each region its label. A region is then set as blur if the main part of it is recovered by the Blur area. A numerical vector of region descriptors and a label are then obtained for each region. These data are the training data used during the supervised learning task, described in a next section.

\section{B. Identification task}

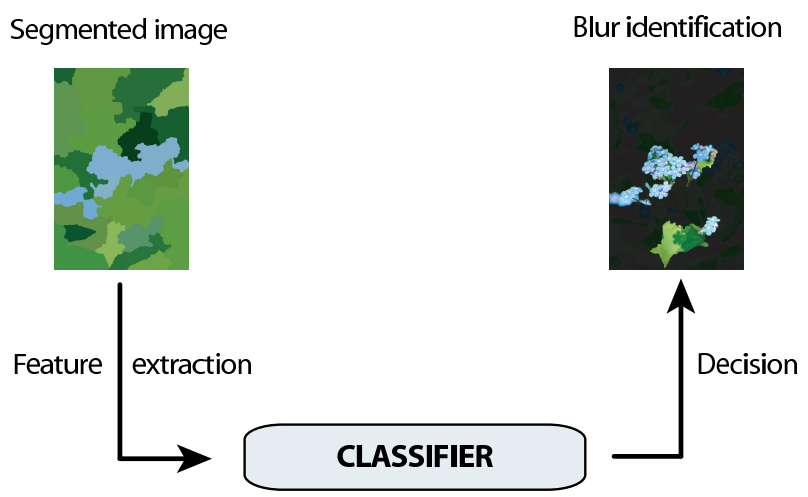

Fig. 4. The identification process.

As shown in figure 4 the identification task is divided in different steps:

- segmentation of the image.

- computation, for each region, of numerical features.

- decision, for each region, of the "Blur / No Blur" label using the classifier.

We have described outlines of the blur identification, we will now present the different steps, beginning by the segmentation task.

\section{SEgMENTATION PROCESS}

This step is an essential low-level one that consists in partitioning the image in visually distinct and homogeneous regions. Several kinds of segmented region may be extracted according to the homogeneity predicates used : color, texture or else semantic criteria. We may notice many approaches[6] and many optimized version of well-known classical algorithms. Objectively, none method seems to be more robust in a general context[7], each of them being more adapted among the content of the image. Our aim here is not to enter in a new 
segmentation debate but to concentrate our efforts on choosing the best segmentation algorithm. First, let then present the required properties of this step we have identified.

\section{A. Segmentation: required properties}

1) Relatively coarse: Small regions may biased the classifier as they reach poor and perturbed information : no decision can be taken on some few pixels.

2) Homogeneous regions: To reach the best learning task as possible it is necessary to segment homogeneous regions in texture and color. Otherwise, to get several and too much different zones agglomerated together may produce a region with numerical biased descriptors.

3) Well separated regions: This is the most important required properties; segmented regions have to recover only one kind of label: Blur or No Blur.

\section{B. The choice of segmentation algorithm}

1) A set of candidates: The segmentation algorithm must integrate as mush as possible these properties and we have introduced an objective protocol in order to select the most adapted algorithm. To that effect, we first have chosen four distinct classical methods covering a large panel of existing approaches : WaterShed [8], Pyramid [9], MeanShift [10] and Histogram [11].

2) Segmentation evaluation: The literature is plenty of segmentation evaluations ([12], [13], [14]) but it is difficult to adapt any protocol to our case. Moreover, to evaluate if the algorithm does not group too much blur and non blur zone in a same region, it seems logical to measure what we may call "mix coefficient" on a set of images.

Given a segmentation $X=\bigcup_{j=1}^{m} X_{j}$ and $X=(B, N B)$ the manual annotation of the image $\mathrm{X}$ - noting $\|A\|$ as the surface in pixels of $A$ - we define the mix parameter as follow:

$$
\delta_{j}=\left\{\begin{array}{l}
1 \text { If } \frac{\min \left(\left\|X_{j} \cap B\right\|,\left\|X_{j} \cap N B\right\|\right)}{\left\|X_{j}\right\|} \geq \alpha \\
0 \text { Else }
\end{array}\right.
$$

$\alpha$ is a threshold ${ }^{2}$ that enables a region to be considered mixed only if the less represented label is at least present at a ratio of $\alpha$.

Then, the "mix coefficient" of one image is done by the formula:

$$
m i x=\frac{\sum_{j=1}^{m} \delta_{j}}{m}
$$

The mix value for one image expresses the percent of regions that are potentially biased, in fact the percent of $n-$ tuple that would be able to disturb the supervised learning.

Also, we will evaluate the confusion matrix $\mathrm{C}$ defined as follow:

$$
C_{(\Lambda, \Theta)}^{j}=\left\{\begin{array}{ll}
0 & \text { if } \\
\left(\left\|X_{j} \cap \Theta\right\|\right. & \text { else }
\end{array}\left(\left\|X_{j} \cap \Lambda\right\|<\left\|X_{j} \cap \Theta\right\|\right)\right.
$$

\footnotetext{
${ }^{2}$ set to 0,02 in this study
}

$C_{(\Lambda, \Theta)}^{j}$ is 0 if the region $X_{j}$ is mainly composed by pixels of class $\Theta$ and represent the number of pixels $\Theta$ otherwise. $\Lambda$ and $\Theta$ take values in set $\{B, N B\}$.

Then, the matrix $C$ is defined by the formula:

$$
C_{(\Lambda, \Theta)}=\frac{\sum_{j=1}^{m} C_{(\Lambda, \Theta)}^{j}}{\|X\|}
$$

For example, $C_{(B, N B)}$ represents the percent of pixels of class $N B$ that are mixed with a region mainly composed by pixels of class $B$. Consequently, the value $C_{(B, N B)}+$ $C_{(N B, B)}$ is the minimum error (in percent of pixels) using the considered segmentation that an identification task may do.

3) Results and conclusion: Tables I and II show the average parameters computed on the training set. Color based approaches are clearly less efficient and do not correspond to our expectations. Also, the two other approaches gave similar results, even if the wathershed one seems more adapted to our case. Therefore, the end of this paper will consider the watershed approach as segmentation algorithm.

These results show than $21 \%$ percent of regions contain Blurry and Non Blurry zones. It intends to the supervised learning that about $20 \%$ of tuples are possibly biased. Also the minimum error as defined before is $4.1 \%$, we may then conclude that if $20 \%$ of regions are mixed few of them do not have a very majority class. Finally, $4.1 \%$ of minimum error (in pixels) is a small value, we may expect a high accuracy.

TABLE I

SEGMENTATION EVALUATION - MIX COEFFICIENT

\begin{tabular}{|c|c|}
\hline Method & Average mix \\
\hline WaterShed & 0.21 \\
Pyramid & 0.25 \\
MeanShift & 0.35 \\
Histogram & 0.36 \\
\hline
\end{tabular}

TABLE II

SEgmentation EVALUATION - CONFUSION MATRICES

\begin{tabular}{|c|c|c|}
\hline WaterShed & B & NB \\
\hline B & 46.1 & 2.2 \\
\hline NB & 1.9 & 49.8 \\
\hline
\end{tabular}

\begin{tabular}{|c|c|c|}
\hline Pyramid & B & NB \\
\hline B & 46.2 & 2.1 \\
\hline NB & 2.3 & 49.4 \\
\hline
\end{tabular}

\begin{tabular}{|c|c|c|}
\hline MeanShift & B & NB \\
\hline B & 43.9 & 4.4 \\
\hline NB & 3.6 & 48.1 \\
\hline
\end{tabular}

\begin{tabular}{|c|c|c|}
\hline Histogram & B & NB \\
\hline B & 44.2 & 4.1 \\
\hline NB & 4.3 & 47.4 \\
\hline
\end{tabular}

\section{REGION DESCRIPTORS}

Our basic idea is that blurry regions are more invariant according to low pass filtering. Moreover, it seems to be difficult to quantify blurry regions with absolute parameters. On the contrary, only relative evolutions between two treatments are able to be characteristic as a blurry region is visually less influenced by Gaussian filtering than a non blurry one. The figure 5 illustrates this behavior. 


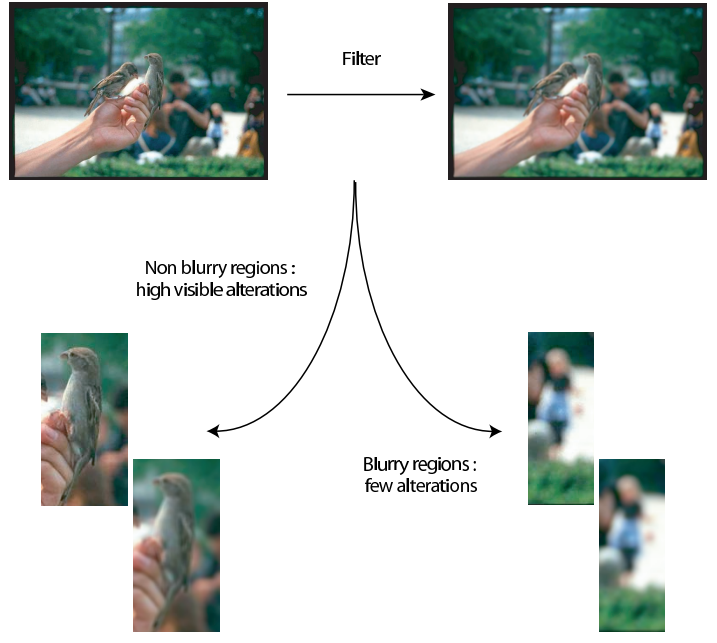

Fig. 5. Filtering and blur

\section{A. Filtering evolution}

Proposed descriptors are then classified into three families

- Evolution of classical moments.

- Evolution of texture descriptors.

- Evolution of high frequencies.

1) Statistical moments: First of all, the first four classical moments are computed on the original image and different low-pass filtered versions (from a $3 \times 3$ to a $9 \times 9$ one).

For each filtered image is notably associated

$$
\frac{\mathcal{M}_{n}^{F}-\mathcal{M}_{n}^{o}}{\mathcal{M}_{n}^{o}}
$$

where $\mathcal{M}_{n}^{o}$ and $\mathcal{M}_{n}^{F}$ are respectively statistical n-order moments of the original image and the filtered one.

2) Textural descriptors: Many studies about texture can be found in the literature, from the co-occurrence matrices to the wavelet transforms. Among these options, we have retained run-length matrices[15] and its color application[16]. More particularly, these parameters suppose to first realize a quantization of the image. So, during our study, we have implemented a quantization based on a k-means algorithm.

Let precise the run-lengths used parameters. This matrix is of size $C \times T$ where $C$ is the number of quantized colors and $T$ is the maximum length in the considered direction. More precisely, a matrix is computed for each region defined by the segmentation process. Let then note $L^{\Theta}[i, j]$ the number of sections of color $i$ and length $j$ in the direction $\Theta$, that is to say that $j$ pixels of entry $i$ in the quantization image are adjacent in the direction $\Theta$. The most characteristic directions are in natural images 0 and 90 degrees, but we compute each direction from 0 to 315 degrees with a 45 degrees step. Among the most discriminate parameters, we have retained the following ones:
- Weight of short sections

$$
\frac{1}{\sum_{i=1}^{C} \sum_{j=1}^{T} L^{\Theta}[i, j]} \sum_{i=1}^{C} \sum_{j=1}^{T} \frac{L^{\Theta}[i, j]}{j^{2}}
$$

- Weight of long sections

$$
\frac{1}{\sum_{i=1}^{C} \sum_{j=1}^{T} L^{\Theta}[i, j]} \sum_{i=1}^{C} \sum_{j=1}^{T} L^{\Theta}[i, j] \times j^{2}
$$

- Inhomogeneity

$$
\frac{1}{\sum_{i=1}^{C} \sum_{j=1}^{T} L^{\Theta}[i, j]} \sum_{i=1}^{C}\left(\sum_{j=1}^{T} L^{\Theta}[i, j]\right)^{2}
$$

Each parameter is computed with $\Theta=0, \Theta=90$ and the minimum and maximum values are retained too, that is to

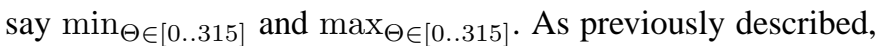
the evolution of each parameter computed on the original image and different filtered versions are added, so that each parameter is no more absolute but relative, improving normally its capability to discriminate blurry to non blurry regions. Objectively, the weight of short sections for example seems to be more influenced by the filtering.

In the same way, we have previously noticed the influence of the quantization step. In order to ponderate it, we have computed on each region the only number of quantized colors and the evolution through the filtering versions, assuming the fact that blurry regions are less textured. Logically, the blurry regions must be less influenced by the filtering too.

3) High frequencies descriptors: Image enhancement and restoration of noised and blurred images methods are common procedures intented to process an image so that the resulting processed image is more suitable. Among the large collections of methods [17], [18], [19], we have retained the Sapiro's approach[20], notably because of its color application. The method consists in an anisotropic diffusion algorithm. Our idea is that the difference between the original and the restored image will be more sensitive in the blurred regions.

Then, we compute first statistical moments of the differences image on each region. The two first moments are noticed to be the more characteristic.

\section{B. Edge ratio}

Visually we may conclude that a blurry region contains less sharp edges than a non blurry one. We then evaluate the edge ratio. Using the classical Prewitt detector[21], we compute, for each region, the percent of pixels that are located on an edge. The threshold was pre-defined for all images and the choice of 150 has been retained for this study.

\section{Point Spread Function}

We choose to implement a PSF estimator based on Lipschitz-Holder exponent[22], [23]. Computed in the wavelet domain, this descriptor is robust to noise and may be defined as how smooth the image is on the considered point. 


\section{NeURAL NETWORK}

Neural network approach is well-adapted to our data and problematic. Other methods[24], [25], [26] like decision tree or support vector machine do not fit precisely the kind of data presented here and give really less efficient results on blur identification.

The neural network classifier is constructed for the features using the back-propagation learning algorithm[27], [28]. This is a supervised classifier. It takes as input a numerical vector of features. The output is the category of the region. The neural network then learns by adjusting its weights as it processes the training data.

\section{EVALUATION}

\section{A. Objective evaluation}

1) Effectiveness measures: To evaluate the ability of this identification in our context, it is necessary to introduce several measures of effectiveness. Let $N$ the number of elements to classify, $f_{i}$ the classifier decision class and $a_{i}$ the ground-truth class ot the $i$-th element. We will consider Blur label as the true instance and NoBlur as the false.

- Overvall efficiency of the classifier

$$
\text { Accuracy }=\frac{\left|\left\{i ; f_{i}=a_{i}\right\}\right|}{N}
$$

- Precision

$$
\text { Precision }=\frac{\mid\left\{i ; f_{i}=a_{i} \& a_{i}=\text { true }\right\} \mid}{\mid\left\{i ; f_{i}=\text { true }\right\} \mid}
$$

- Recall

$$
\text { Recall }=\frac{\mid\left\{i ; f_{i}=a_{i} \& a_{i}=\text { true }\right\} \mid}{\mid\left\{i ; a_{i}=\text { true }\right\} \mid}
$$

2) Cross Validation: To estimate the final efficiency of a method, we wonder an estimation method with low bias, low variance and handling over-fitting. Between well-known accuracy estimation methods, we choose cross-validation [29] as it is intelligible, simple to implement and efficient. We have selected a 10-Fold cross-validation: the set is split into 10 random partitions. In each fold, 9 are used as the training set and 1 used as the test set. We then measure score only on the test. The averaged iteration score over all 10 folds is presented.

3) Regions and pixels: We distinguish two different results:

- by region - Measures are obtained classically : we compute accuracy, precision and recall as described below.

- by pixel - Accuracy, precision and recall are obtained using the confusion matrix computed in percent of pixels grouping all regions in image.

\section{B. Results}

These results, presented in tables III and IV, includes more than 100 various images with blurry and non-blurry regions. The training and validation set size were together about 1800 regions.
TABLE III

CROSS-VALIDATION RESULTS, BY REGION

\begin{tabular}{|c|c|}
\hline Precision & 0.89 \\
\hline Recall & 0.82 \\
\hline Accuracy & 0.88 \\
\hline
\end{tabular}

TABLE IV

CROSS-VALIDATION RESULTS, BY PIXEL

\begin{tabular}{|c|c|}
\hline Precision & 0.91 \\
\hline Recall & 0.84 \\
\hline Accuracy & 0.90 \\
\hline
\end{tabular}

\section{Visual examples}

Figure 6 shows some examples of blur identification. Original images are presented on the left and result images - blurry region were colored in black - presented on the right.

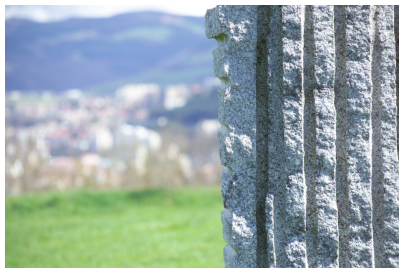

(a)

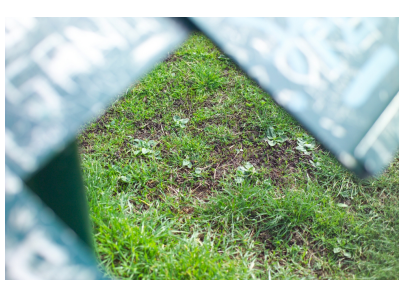

(c)

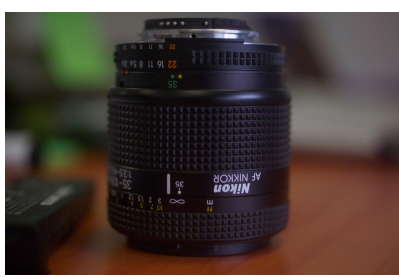

(e)

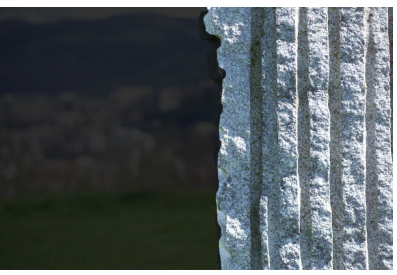

(b)

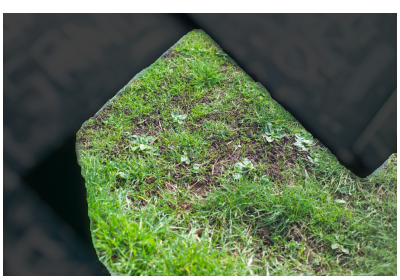

(d)

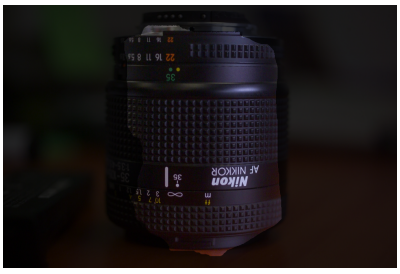

(f)
Fig. 6. Some blur identification.

\section{Objective and subjective Results: discussion}

Visually, as shown in these examples, we retrieve the numerical measures of accuracy : the main majority of pixels and regions are well classified. These examples are also representative of the misclassified pixels : they are generally located on the borders between the blurry and the sharp zones. More the "edge" between these two zones is smooth, more identification is penalized. Logically, we can notice that identification is more difficult when focused objects are not clearly in a different depth than background elements. We may 
assume that, on this kind of images, the main problematic is the segmentation process itself.

Blur identification, measured in pixels or in regions, is done with a precision and a recall about $90 \%$ and $80 \%$. It proves the effectiveness of our scheme and encourages to optimize the different steps of it. Also, results counting pixels or counting regions are similar, even if accuracy measures are higher by pixels: the misclassified regions are not the biggest ones but globally the smaller ones, where the decision is objectively more randomized.

\section{CONCLUSION AND FUTURE WORK}

We have presented a new scheme for achieving blur identification. The first experiments indicate that the introduced decision neural network permits to reach our goal quite accurately. The overall process we have propose in this study is a new strategy that may use in many image processing tools. In deed, to improve their effectivity to integrate this low-level content, precisely the focused objects, is a way to explore. For example, this meta-data may be used directly to many tasks realized by any amateur photograph who desires to add modifications or effects to its own images.

Interesting directions for future works include to improve the segmentation step. To avoid the bias generated by the partition it needs to develop a supervised segmentation algorithm taking account blur estimators. Then it would be possible to get a partition reducing considerably the combining of blurry and sharp area in same region.

Finally, in order to generalize this identification to any kind of blur it will be necessary to model each of them and to propose new numerical descriptors. In this context, spatial relationship and coherence between regions would be new parameters to integrate in the scheme. In deed, the region neighborhood may influence the blur identification for the region itself.

\section{REFERENCES}

[1] H.-C. Lee, "Review of image-blur models in a photographic system using principles of optics," Optical Engineering, vol. 29, no. 5, pp. 405-421, 1990.

[2] A. E. Savakis and H. J. Trussell, "On the accuracy of psf representation in image restoration," IEEE Trans. Image Processing, vol. 2, pp. 252259, 1993.

[3] Y. Xiong and S.A. Shafer, "Depth from focusing and defocusing," in DARPA93, 1993, pp. 967-.

[4] D. Ziou, "Passive depth from defocus using a spatial domain approach," in $I C C V, 1998$, pp. 799-804.

[5] MA. Georgeson and ST. Hammett, "Seeing blur: 'motion sharpening' without motion.," Proc Biol Sci, vol. 269, no. 1499, pp. 1429-1434, 2002.

[6] N. R. Pal and S. K. Pal, "A review on image segmentation techniques," Pattern Recognition, vol. 26, pp. 1277-1294, 1993.

[7] J. P. Cocquerez and S. Philipp, Analyse d'images: filtrage et segmentation, Masson, 1995.

[8] M.C. de Andrade, G. Bertrand, and A.A. Araújo, "An attribute-based image segmentation method," Materials Research, vol. 2, no. 3, pp. 145-151, 1999.

[9] Jrôme Da Rugna and Hubert Konik, "Color coarse segmentation and regions selection for similar images retrieval," in Color in Graphics, Image and Vision. 2002, pp. 241-244, Society for Imaging Science and Technology.
[10] D. Comaniciu and P. Meer, "Mean shift: a robust approach toward feature space analysis," IEEE Transactions on Pattern Analysis and Machine Intelligence, vol. 24, pp. 1-18, 2002.

[11] H. Cheng, "A hierarchical approach to color image segmentation using homogeneity," IEEE Trans. on Image Processing, vol. 9, no. 12, pp. 2071-2082, december 2000.

[12] Y.J. Zhang, "Evaluation and comparison of different segmentation algorithms," Pattern Recognition Letters, vol. 18, pp. 963-974, 1997.

[13] M. Borsotti, P. Campadelli, and R. Schettini, "Quantitative evaluation of color image segmentation results," Pattern Recognition Letters, vol. 19, pp. 741-747, 1998.

[14] P. Correia and F. Pereira, "Standalone objective segmentation quality evaluation," Journal on Applied Signal Processing, vol. 2002, no. 4, pp. 389-400, 2002.

[15] M. Galloway, "Texture analysis using gray-level run length," in Computer graphics and image processing, 1974, vol. 4, pp. 172-199.

[16] C. Vertan, M. Ciuc, V. Buzuloiu, and C. Fernandez-Maloigne, "Compact color-texture run-length description for ornamental stones recognition and indexing," in The Hyperion Scientific Journal, 2002, vol. 3A, pp. 69-75.

[17] J. A. Silva Centeno and V. Haertel, "An adaptive image enhancement algorithm," in Pattern Recognition, 1997, vol. 30, pp. 1183-1189.

[18] K. Deguchi, T. Izumitani, and H. Hontani, "Detection and enhancement of line structures in an image by anisotropic diffusion," in Pattern Recognition Letters, 2002, vol. 23, pp. 1399-1405.

[19] L. M. Kennedy and M. Basu, "Application of projection pursuit learning to boundary detection and deblurring in images," in Pattern Recognition, 2000, vol. 33, pp. 2019-2031.

[20] G. Sapiro and D. L. Ringach, "Anisotropic diffusion of multivalued images to color filtering," in IEEE Transactions on Image Processing, 1996, vol. 5, pp. 1582-1585.

[21] A. Koschan and M. Abidi, "Detection and classification of edges in color images," Signal Processing Magazine, vol. 22, no. 1, pp. 64-73, 2005.

[22] F. Rooms, M. Ronsse, A. Pizurica, and W. Philips, "Psf estimation with applications in autofocus and image restoration," in 3rd IEEE Benelux Signal Processing Symposium, 2002, pp. 13-16.

[23] S. Jaffard, "Exposants de holder en des points donnés et coefficients d'ondelettes," C. R. Acad. Sc., vol. 308, pp. 79-81, 1989.

[24] J. R. Quinlan, "Improved use of continuous attributes in c4.5," Journal of Artificial Intelligence Research, vol. 4, pp. 77-90, 1996.

[25] Tom M. Mitchell, Machine Learning, McGraw-Hill, New York, 1997.

[26] Thorsten Joachims, "Making large-scale svm learning practical. advances in kernel methods - support vector learning," 1999.

[27] J. E. Dayhoff, Neural Network Architectures An Introduction, VNR Press, 1990.

[28] S. Garner, "Weka: The waikato environment for knowledge analysis," 1995.

[29] Ron Kohavi, "A study of cross-validation and bootstrap for accuracy estimation and model selection," in IJCAI, 1995, pp. 1137-1145. 